\title{
3D $h p$-Adaptive Finite Element Simulations of Bend, Step, and Magic-T Electromagnetic Waveguide Structures
}

I. Gomez-Revuelto ${ }^{a}$, L. E. Garcia-Castillo ${ }^{b}$, S. Llorente-Romano $^{b}$, and D. $\operatorname{Pardo}^{c, d}$

${ }^{a}$ Departamento de Ingeniería Audiovisual y Comunicaciones. Universidad Politécnica de Madrid, Madrid, Spain.

${ }^{b}$ Departamento de Teoría de la Señal y Comunicaciones. Universidad Carlos III de Madrid.

${ }^{c}$ Department of Applied Mathematics, Statistics, and Operational Research, University of the Basque Country (UPV/EHU), Leioa, Spain.

${ }^{d}$ IKERBASQUE, Basque Foundation for Science, Bilbao, Spain.

\begin{abstract}
Metallic rectangular waveguides are often the preferred choice on telecommunication systems and medical equipment working on the upper microwave and millimeter wave frequency bands due to the simplicity of its geometry, low losses, and the capacity to handle high powers. Waveguide translational symmetry is interrupted by the unavoidable presence of bends, transitions, and junctions, among others. This paper employs a $3 \mathrm{D} h p$ self-adaptive grid-refinement finite element strategy for the solution of these relevant electromagnetic waveguide problems. These structures often incorporates dielectrics, metallic screws, round corners, and so on, which may facilitate its construction or improve its design, but significantly difficult its modeling when employing semi-analytical techniques. The $h p$-adaptive finite element method enables accurate modeling of these structures even in the presence of complex materials and geometries. Numerical results demonstrate the suit-
\end{abstract}

${ }^{*}$ The work reported in this paper was funded by the Spanish Ministry of Science and Innovation under the projects TEC2010-18175/TCM and MTM2010-16511. The fourth author was also funded by the Laboratory of Mathematics (UFI11/52) at UPV/EHU and the CYTED 2011 Project with reference P711RT0278.

**E-mail addresses: igomez@diac.upm.es, [luise,sllorente]@tsc.uc3m.es,dzubiaur@gmail.com. 
ability of the $h p$-adaptive method for modeling these waveguide structures, delivering errors below $0.5 \%$ with a limited number of unknowns. Solutions of waveguide problems obtained with the self-adaptive $h p$-FEM are of comparable accuracy to those obtained with semi-analytical techniques such as the Mode Matching method, for problems where the latest methods can be applied. At the same time, the $h p$-adaptive FEM enables accurate modeling of more complex waveguide structures.

Keywords: Finite Element Method (FEM), hp-adaptivity, electromagnetic waveguides.

\section{Introduction}

Electromagnetic (EM) waveguides are of great importance for our daily lives, since they can be found in a variety of devices, including communication systems, radars, satellites, and medical equipment. In this paper, we focus on specific types of passive rectangular metallic waveguides.

The translational symmetry of the waveguide is unavoidably interrupted in practice with the presence of bends or transitions as those considered in our applications. In these type of structures, the goal is to achieve very low levels of energy reflected back to the transmitter. Thus, the amount of power delivered to the receiver is maximized. It is worth noting that refelected energy can also affect the transmitter.

Rectangular waveguides (as well as many other waveguides) operate in the monomode region, that is, the excitation frequency is such that when the ports are placed at a certain distance from its center, then only one propagating mode (the so-called $\mathrm{TE}_{10}$ mode) is present with a non-negligible amplitude at the ports. All other modes are evanescent, and their amplitude quickly diminish as the distance from the waveguide discontinuity increases. This implies that the EM field at the ports can be characterized by simply measuring the amount of energy of the propagating mode that is reflected to all ports of the structure (including the excitation port). Theses quantities normalized by the incident energy ("incident power wave") are related by the so-called scattering parameters, also known as S-parameters. More generally, we denote by $S_{i j}$ to the S-parameter measured at port $j$ when the $i$-th port is excited. Specifically, $\left|S_{i i}\right|^{2}$ represents the ratio between the mean powers of the reflected and incident waves. The argument of $S_{i i}$ represents the phase shift between the reflected and incident waves. Analogously, $\left|S_{j i}\right|^{2}$, 
$j \neq i$ relates the power relation between the transmitted wave at port- $j$ and the incident wave at port- $i$; the argument of $S_{j i}$ gives the phase shift between transmitted and incident waves. The corresponding $[S]$ matrix with entries $S_{i j}$ is the so-called scattering matrix. The reciprocity of the passive waveguides makes $[S]$ matrix to be symmetric (not Hermitian). The geometrical symmetries of the structure (when present) add extra equality relations between the $S_{i j}$ coefficients, as it will be clear later.

Thus, the main objective of the simulations for structures such as bends and transitions between waveguides is to estimate the S-parameters. If the results are unsatisfactory according to some specific energy transmission/reflection pattern, further design modifications on the waveguide structure can be realized; for example, by altering the original geometry or by introducing small dielectrics in the interior. Sometimes, a number of transitions are combined in order to perform a more sophisticated electric function. That is the case of cavity filters in which a number of resonance cavities are coupled to each other (and to the input and output waveguides) with transitions as the one analyzed in this paper. The accurate characterization of each transition, not only in terms of reflected and transmited power but also in terms of the phases of the waves scattered by the transitions, are crucial.

Sophisticated power divider and power combiner electrical functions can be accomplished with multi-port junctions. As suggested by its name, power dividers/combiners are used to either divide or combine the power of a given EM signal and they are employed, among other applications, in RF \& Microwave front-ends, and various types of antennas. One of such power dividers is the so-called Magic-T (also known as magic tee), first described in [1]. It consists of a "combination" of two orthogonal T-shaped junctions, one in the magnetic H-plane and one in the electric E-plane. Therefore, when an input signal is injected into the H-port ${ }^{1}$ (port 3 ), the output signal becomes equally distributed between ports 1 and 2 and will be in phase. When an input signal is injected into the E-port (port 4), the output signal also becomes equally distributed between ports 3 and 4, but this time the output signals will have opposite sign (180 degrees out of phase). Its name comes from its magical electrical properties. Namely, when ports 3 and 4 are simultaneously matched, that is, when the structure is designed in such a way that the energy reflected at these ports is null, then one can show that ports 1 and 2

\footnotetext{
${ }^{1}$ The ports are the apertures at the end of the waveguides.
} 
become "magically" matched and decoupled. Thus, the desired $S$ coefficients in magnitude are:

$$
\left[\left|S_{i j}\right|\right]=\frac{1}{\sqrt{2}}\left[\begin{array}{cccc}
0 & 0 & 1 & -1 \\
0 & 0 & 1 & 1 \\
1 & 1 & 0 & 0 \\
-1 & 1 & 0 & 0
\end{array}\right]
$$

The isolation between ports 3 and 4 comes simply by the properties of the E-plane and H-plane T-junctions. However, as mentioned previously, the isolation between ports 1 and 2 requires the compensation of the magic- $\mathrm{T}$ so ports 3 and 4 are simultaneously matched. This can be accomplished by the use of internal posts or other techniques without disturbing the symmetry of the structure (c.f., [2]). Due to design limitations (e.g., geometry, size, construction materials and imperfections), it is impossible in practice to achieve the above scattering pattern, and suboptimal designs need to be developed. In this context, the use of numerical simulations is essential both to design improved waveguides under certain restrictions as well as to evaluate the performance of a given waveguide configuration.

Several computational techniques can be used for the analysis of bends, transitions, and junctions as the Magic-T waveguide structure. First, we have analytic (or semi-analytic) techniques such as the Mode Matching (MM) method (c.f., $[3,4]$ ), which are computationally very efficient, and they can be employed to design several devices with given specifications using optimization procedures $[5,6]$. These methods have severe limitations in terms of the geometries they can handle. Thus, hybrid extensions as those of $[7,8,9,10]$ have been developed in the last years. They overcome many of the limitations of pure analytic (or semi-analytic) techniques but still need "separable" geometries, which limits the type of geometries they can handle. Specifically, they cannot support the presence of arbitrary objects such as screws or curved geometries that can appear as a result of the waveguide construction and manufacturing. Thus, the use of pure numerical methods that can analyze the structure as a whole, taking into account the effect of round corners due to finite radii of milling tools, the influence/sensibility to position of tuning elements (posts, screws, etc) not oriented in the coordinate axis, losses, and so on, is preferred.

The main advantage of pure discretization methods such as finite element methods (FEM) and Integral Equation (IE) approaches is their flexibility 
to model arbitrary materials and geometries. Moreover, even for devices designed with semi-analytic methods, they need to be finally validated with a pure numerical technique that can analyze the structure as a whole, taking into account the effect of round corners due to finite radii of milling tools, the influence/sensibility to position of tuning elements (posts, screws, etc), losses, and so on. In this context, the FEM has demonstrated to be a powerful and versatile tool. However, high accuracy is not always easy to achieve for conventional FEM when dealing with field singularities, high-contrast material data, etc. Thus, semi-analytic results (when available) are typically considered as a reference for engineering analysis.

In this work, the use of a highly-accurate three-dimensional (3D) fully automatic $h p$-adaptive FEM for the characterization of waveguide components is proposed. This method combines the geometrical flexibility of a FEM with an accuracy that is often superior to that provided by semi-analytical methods, as it will be shown in Section 4. Such a high-accuracy delivered by the $h p$-FEM is due to the exponential convergence of the error on optimally designed $h p$-meshes. These meshes adapt to the problem of interest, and are produced automatically (without any user-interaction) by the $h p$-adaptive strategy. This grid-refinement strategy efficiently deals with different types of singularities. Moreover, the dispersion (pollution) error is also automatically minimized by increasing the polynomial order of approximation (see $[11,12]$ ), and therefore, the method is also capable of solving efficiently high-frequency waveguide problems.

This paper constitutes an extension of our previous 2D paper [13] to the case of 3D waveguides. The level of complexity in 3D (in both, mathematical and computational senses) of the $h p$ FE method, and specifically of the $h p$ adaptivity, is several orders of magnitude higher than in 2D. This is due, among other reasons, to the higher number of "choices" available to decide the next $h p$-mesh at each step of the adaptivity. Also, it is due to the (higher) computational order of complexity with polynomial order $p$ involved in the computations. In addition, a much larger number of unknowns are usually involved in 3D problems when compared with 2D problems.

The particular $h p$-adaptive algorithm used in this paper builds upon previous versions developed by L. Demkowicz and his collaborators $[14,15,16$, $17,18]$. In the version used in this work, we fixed a number of errors and implementation problems, we increased its performance for realistic 3D computations, and we implemented proper post-processing schemes to compute the S-matrix. 
The paper is organized as follows. Section 2 describes the mathematical formulation of the problem. Then, the $h p$-method including the selfadaptive refinement strategy is detailed in Section 3. Section 4 outlines the main results for several waveguide components obtained with the $h p$-adaptive method. Finally, some conclusions are depicted in Section 5.

\section{Mathematical Formulation}

The EM phenomena are governed by a set of four first order partial differential equations (PDEs) known as Maxwell's equations. For a time-harmonic nonzero frequency excitation $(\omega \neq 0)$, two of those equations (the so-called Gauss's laws) become redundant, and the remaining two equations (Ampere's and Faraday's laws) along with the corresponding boundary conditions provide a unique solution for the EM field:

$$
\begin{array}{cccc}
\boldsymbol{\nabla} \times \mathbf{E}= & -j \omega \mu \mathbf{H}+\mathbf{J}^{i m p} & & \text { (Faraday's law) } \\
\boldsymbol{\nabla} \times \mathbf{H}= & j \omega \varepsilon \mathbf{E} & & \text { (Ampere's law) }
\end{array}
$$

In the above equations, $\mathbf{E}$ and $\mathbf{H}$ stand for the electric and magnetic field, respectively, $j=\sqrt{-1}$ is the imaginary unit, $\epsilon$ and $\mu$ are the electric permittivity and permeability of the medium, respectively, and $\mathbf{J}^{i m p}$ is the impressed volumetric electric current, which is zero in our case.

The above system of two first order PDE's can be further simplified to a single second order PDE in terms of magnetic field $\mathbf{H}$ by taking the curl of Ampere's law pre-multiplied by $1 / \epsilon$ and using Faraday's law to obtain the so called reduced wave equation:

$$
\boldsymbol{\nabla} \times \frac{1}{\varepsilon} \boldsymbol{\nabla} \times \mathbf{H}-\omega^{2} \mu \mathbf{H}=0
$$

Assuming $\mathrm{TE}_{10}$ mode excitation and excitation frequency in the monomode region, the following boundary condition can be used at the ports, namely:

$$
\hat{\mathbf{n}} \times \frac{1}{\varepsilon} \boldsymbol{\nabla} \times \mathbf{H}+j \frac{\omega^{2} \mu}{\beta_{10}} \hat{\mathbf{n}} \times \hat{\mathbf{n}} \times \mathbf{H}=\mathbf{U}^{\text {in }} \text {, at the port boundaries }\left(\Gamma_{p}\right)
$$

where $\hat{\mathbf{n}}$ is the unit outward vector normal to $\Gamma, \beta_{10}$ is the phase constant of the $\mathrm{TE}_{10}$, and $\mathbf{U}^{\mathrm{in}}$ is defined as

$$
\mathbf{U}^{\text {in }}=2 j \frac{\omega^{2} \mu}{\beta_{10}} \hat{\mathbf{n}} \times \hat{\mathbf{n}} \times \mathbf{H}^{\text {in }}
$$


with $\mathbf{H}^{\text {in }}$ being the incident magnetic field at the port. When $\mathbf{H}^{\text {in }}=\mathbf{0}$, the above boundary conditions at the ports (3) become absorbing boundary conditions for the monomode propagation case. The remaining modes are evanescent when in the monomode region, and they quickly diminish in amplitude as they travel towards the ports. Thus, it is necessary to truncate the FEM domain at a certain distance from the waveguide discontinuity so the amplitude of the evanescent modes becomes negligible. The waveguide walls are modelled with perfect electric boundary conditions:

$$
\hat{\mathbf{n}} \times \frac{1}{\varepsilon} \nabla \times \mathbf{H}=0, \quad \text { at perfect electric conductors }\left(\Gamma_{N}\right)
$$

The FEM variational formulation is obtained by multiplying (2) by a test function $\mathbf{F} \in \mathbf{H}(\operatorname{curl}, \Omega)$, integrating by parts, and incorporating the boundary conditions to obtain:

$$
\begin{aligned}
& \int(\boldsymbol{\nabla} \times \mathbf{F}) \cdot\left(\frac{1}{\varepsilon} \nabla \times \mathbf{H}\right) d \Omega-\omega^{2} \int \mathbf{F} \cdot \mu \mathbf{H} d \Omega \\
+ & j \frac{\omega^{2} \mu}{\beta_{10}} \int_{\sum_{\Gamma_{p}^{i}}}(\hat{\mathbf{n}} \times \mathbf{F}) \cdot(\hat{\mathbf{n}} \times \mathbf{H}) d \Gamma=2 j \frac{\omega^{2} \mu}{\beta_{10}} \int_{\Gamma_{p}^{\mathrm{in}}}(\hat{\mathbf{n}} \times \mathbf{F}) \cdot\left(\hat{\mathbf{n}} \times \mathbf{H}^{\mathrm{in}}\right) d \Gamma .
\end{aligned}
$$

We note that the above formulation is given in terms of the magnetic field, although a dual formulation in terms of the electric field can also be derived in a similar way.

\section{3. $h p$ Finite Elements and Automatic Adaptivity}

With each finite element, we associate element size $h$ and order of approximation $p$. In the $h$-adaptive version of FEM, element size $h$ may vary from element to element, while order of approximation $p$ is fixed (usually $p=1,2$ ). In the $p$-adaptive version of the FEM, $p$ may vary locally, while $h$ remains constant throughout the adaptive procedure. Finally, a true $h p$-adaptive version of the FEM allows for local variations of both $h$ and $p$.

The $h p$-FEM constitutes a flexible method suitable for solutions containing both smooth and non-smooth components in different areas of the computational domain $[18,19]$. Solutions incorporating smooth components and a variety of singularities are typical in waveguide applications, and thus, the $h p$-FEM is especially well-suited for these applications. While the spectral $p$-FEM provides accurate approximations of the smooth components of the 
solution and minimizes the dispersion error in the case of wave propagation problems (see $[11,12,20]$ ), the $h$-FEM is intended to accurately approximate singular solutions, as it occurs in the case of re-entrant corners in EM or in areas of the computational domain where three or more materials meet at a point.

To determine an optimal distribution of element size $h$ and polynomial order of approximation $p$, we shall employ a self-adaptive refinement strategy based on the iterative scheme described in [16, 18]. At each step, given an arbitrary $h p$-grid, we first perform a global and uniform $h p$-refinement to obtain the $h / 2, p+1$-grid. Second, we approximate the error function in the $h p$-grid by evaluating the difference between the solutions associated to the $h p$-and $h / 2, p+1$-grids. If the error exceeds a user-prescribed tolerance error, then we project the error function to guide optimal refinements over the $h p$-grid, and we iterate the process. Once the prescribed tolerance error has been met, we deliver the $h / 2, p+1$-grid as the ultimate solution of the problem. This three-dimensional refinement strategy has been proved to be efficient, robust, and highly accurate [18] in many applications.

For optimal selection between $h$ and $p$ refinements, we utilize the projectionbased interpolation (see $[21,22]$ ) operators to project the solution associated to the $h / 2, p+1$-grid into a sequence of coarser grids containing more unknowns than those of the $h p$-grid, and we compare results of those projections to determine the one providing the largest error decrease rate per added unknown. To evaluate the error of each projected solution, we employ the energy norm. To make this optimization problem tractable from the computational point of view, we first determine optimal refinements for edges, then for faces, and finally for element interiors. Critical to the success of this projection based approach is the commutativity of the so-called de Rham diagram, which is used to ensure stability and convergence of the final formulation, avoiding the appearance of spurious (unphysical) modes. The simulator utilizes hexahedral edge (Nedelec) elements of variable order of approximation to discretize $\mathbf{H}$ (curl).

Our $h p$-adaptive FEM supports local anisotropic refinements in both $h$ and $p$. Support of anisotropic refinements dramatically increases the implementation complexity, but it is essential to achieve optimal convergence rates in the case of edge singularities or in the presence of boundary layers. Both situations occur in our problems of interest. To ensure continuity, we enforce the 1-irregularity rule and use the constrained approximation [23].

As mentioned above, the main advantage of the $h p$-FEM resides on the 
proof (see $[24,25,26,27,28,29,30])$ showing that it can achieve exponential convergence for elliptic problems with a piecewise analytic solution, whereas $h$ - or $p$-FEM converge at best algebraically. To attain that goal, we have further extended the existing $h p$-adaptive strategy for the case of waveguide problems.

For the analysis of the waveguide structures, we have implemented impedance boundary conditions as well as several postprocessing routines for computing the S-parameters, graphics, and so on. We have also fixed a few problems and accelerated a number of key features within the software such as the computation of the projections during the $h p$-adaptivity.

\section{Numerical Results}

Numerical results of several waveguide components obtained with the $h p$-adaptive method are shown in the following.

\subsection{Cascade of H-plane and E-plane right angle bends}

This structure consists of the "composition/cascade" of one E-plane bend and one H-plane bend. It can be found on systems with severe restrictions in terms of space as it happens in satellite systems and electronic warfare airbone systems. Both bends are right angle bends. E-plane and H-plane bends are basically two-dimensional bends, where the bend occurs over a plane. Depending of whether the plane contains the electric field or the magnetic field of the $\mathrm{TE}_{10}$ mode, the bend is named E-plane bend or H-plane bend, respectively. The geometry of the structure is depicted in Fig. 1. The structure is analyzed by exciting port 1 and computing $S_{11}$ and $S_{21}$. From the properties of $S$-parameters for a reciprocal lossles two-port network, it is inferred that $S_{21}=S_{12}$ and $\left|S_{11}\right|=\left|S_{22}\right|$. Due to the interaction between the higher modes of the two bends, there is some error if the S-parameters are obtained as the cascade of two bends instead of analyzing the two bends at once. This error decays exponentially with the distance between the bends (parameter $d$ in our analysis). Thus, one should analyze it as a unique three dimensional structure rather than as two separate two-dimensional bends.

For illustration purposes, we analyze two cases with different distances between the bend discontinuities. For the first case, the distance between the bends is selected as $d=0.5 a$. For that distance $d$, it can be estimated that the error in the S-parameters is below $-50 \mathrm{~dB}$ and can be considered negligible in many applications. The second case is selected to have zero 
separation between the bends. This case is the worst in terms of mutual interactions between the bends; the error is still relatively small in practice though. However, the case $d=0$ is very illustrative of the performance of the adaptivity when the two edge singularities collapse.

The initial meshes are displayed in Fig. 2(a) and Fig. 3(a). Note that initial grids consist of very coarse meshes that have been selected in order to assess the robustness of the $h p$ strategy in context of real engineering analysis, in which the initial mesh has to be as coarse as possible to simplify the mesh generation process.

The convergence history (up to an energy error below $0.1 \%$ ) achieved with $h p$ adaptivity for $d=0.5 a, 0$ are displayed in Fig. 4(a) and Fig. 4(b), respectively. A log scale for the energy error (in percent of the energy norm) in the ordinate axis is used while a scale corresponding to $N_{\text {dof }}^{1 / 5}$ is employed in the abscissa axis. This is the type of plot that will be used in the remainder of the paper. Note that the abscissas scale corresponds to $N_{\text {dof }}^{1 / 5}$ while abscissas axis tics should be read as $N_{\text {dof }}$ in the plots. According to [29] and references therein, an approximate straight line should appear with such scales (i.e., corresponding to a behavior of the errror as error $=C \exp \left(-\alpha N_{\text {dof }}^{1 / 5}=\right.$ being $C$ and $\alpha$ constants), indicating the theoretical exponential convergence that can be achieved with an optimal $h p$ adaptive strategy after an initial pre-asymptotic regime, which is the result observed in the figure. It is also observed how the convergence is pretty much insensitive to parameter $d$ illustrating the robustness of the automatic adaptivity algorithm.

When comparing 3D convergence shown in Fig. 4(a) and Fig. 4(b) with the behavior of the adaptivity in $2 \mathrm{D}$ for a simple bend analyzed as a $2 \mathrm{D}$ structure, we observe a similar convergence behavior. However, the number of steps of the adaptivity is larger for 3D than for 2D (around 50\% more). Also, in agreement with the theory, the exponent of $N_{\text {dof }}$ is $1 / 3$ in $2 \mathrm{D}$ in contrast with $1 / 5$ shown above for $3 \mathrm{D}$. Then, despite of the good job performed by the adaptivity in achieving exponential convergence, the rate of increment of the number of degrees of freedom to get a given decrement in the error is noticeably higher in $3 \mathrm{D}$ than in $2 \mathrm{D}$. The above statements are pretty general and hold also for other waveguide structures. In practice, for relative energy norm errors between $1 \%$ and $0.1 \%$, the number of unknowns for the $3 \mathrm{D}$ case are between one and two orders of magnitude larger than in 2D. In addition, the connectivity between degrees of freedom in 3D is higher than in $2 \mathrm{D}$ affecting the performance of the algebraic solvers. 


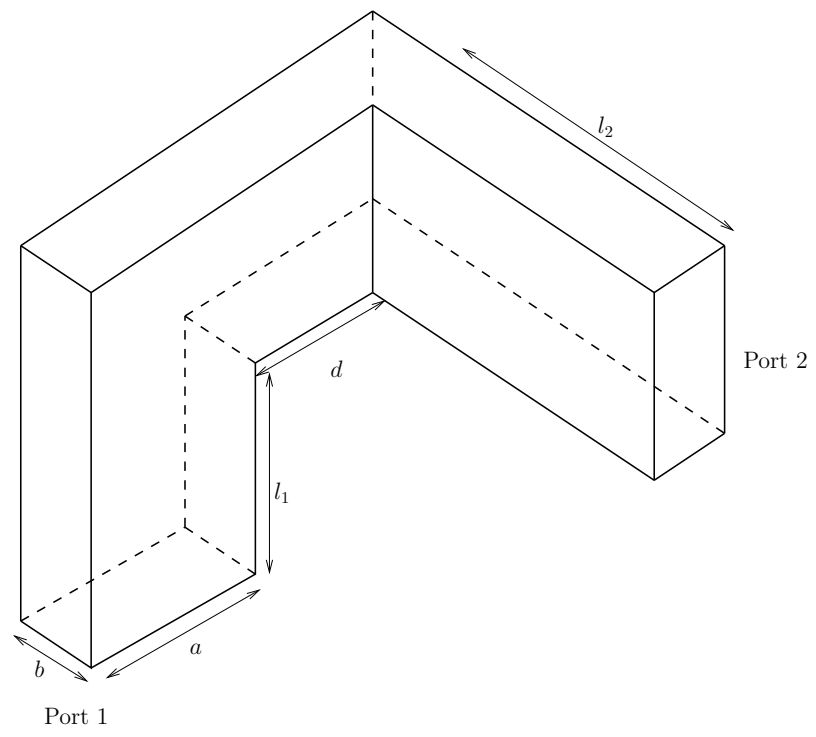

Figure 1: Cascade of H-plane and E-plane bends $\left(b=a / 2, l_{1}=l_{2}=1.5 a\right)$

The final meshes delivered by the $h p$-adaptivity (for an error level around $0.1 \%$ are displayed in Fig. 2(b) Fig. 3(b). It is observed an increase of polynomial order $p$ on those regions where the solution is smooth (i.e., away from the junctions), while intensive $h$-refinements occur close to edge and vertices where the field changes abruptly (field singularities are often present on those regions).

An illustration of the magnetic field in both structures can be seen in Fig. 5; specifically, the real part of the z-component. The scattering parameters computed with the $h p$-FEM are compared with those obtained with a Mode Matching (MM) technique. Only four significant digits are shown in the table as the MM results are presumed to have no more than four digits of accuracy. The results are shown in Table 1 and Table 2. A very good agreement is observed. In this context, we remark that MM is a semi-analytic technique that is typically considered as a reference for the engineering analysis of these structures. Obviously, pure numerical methods as the one proposed here is able to model arbitrary materials and geometries while MM is not. 


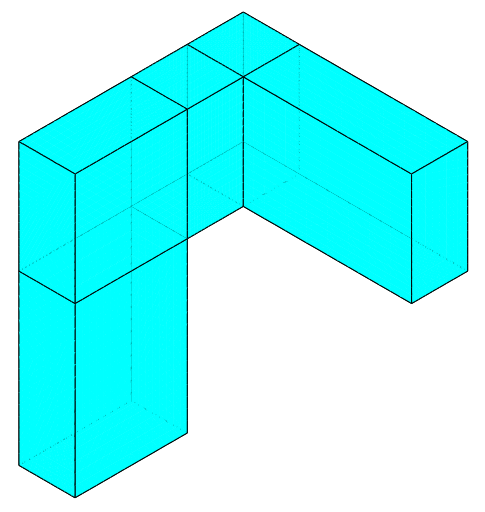

(a) Initial mesh

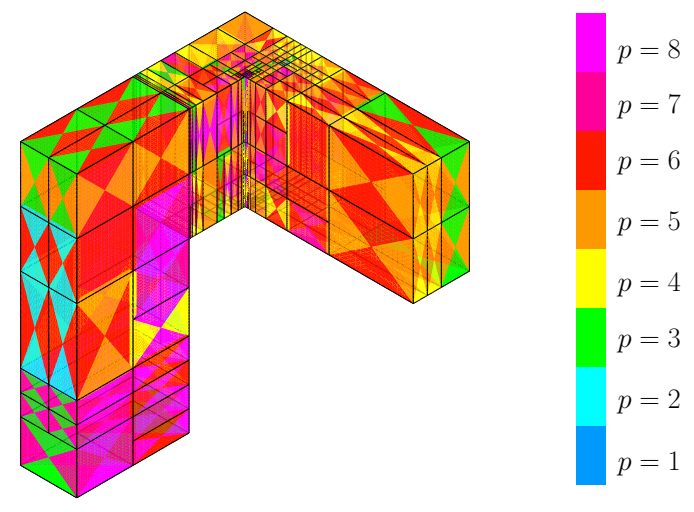

(b) Final mesh

Figure 2: Meshes of the cascade of H-plane and E-plane bends when $d=0.5 a$. Different colors indicate different polynomials orders of approximation.

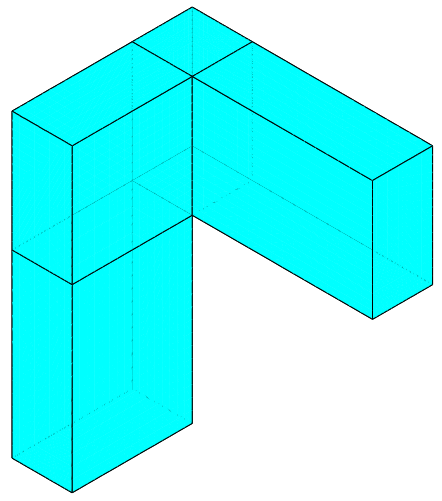

(a) Initial mesh

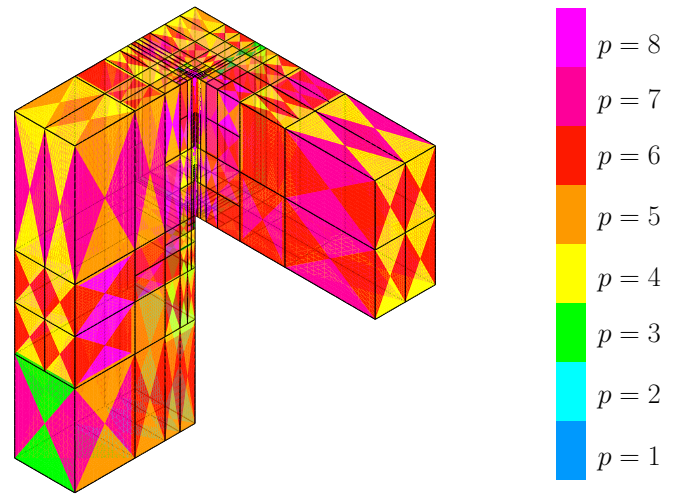

(b) Final mesh

Figure 3: Meshes of the cascade of H-plane and E-plane bends when $d=0$. Different colors indicate different polynomials orders of approximation. 


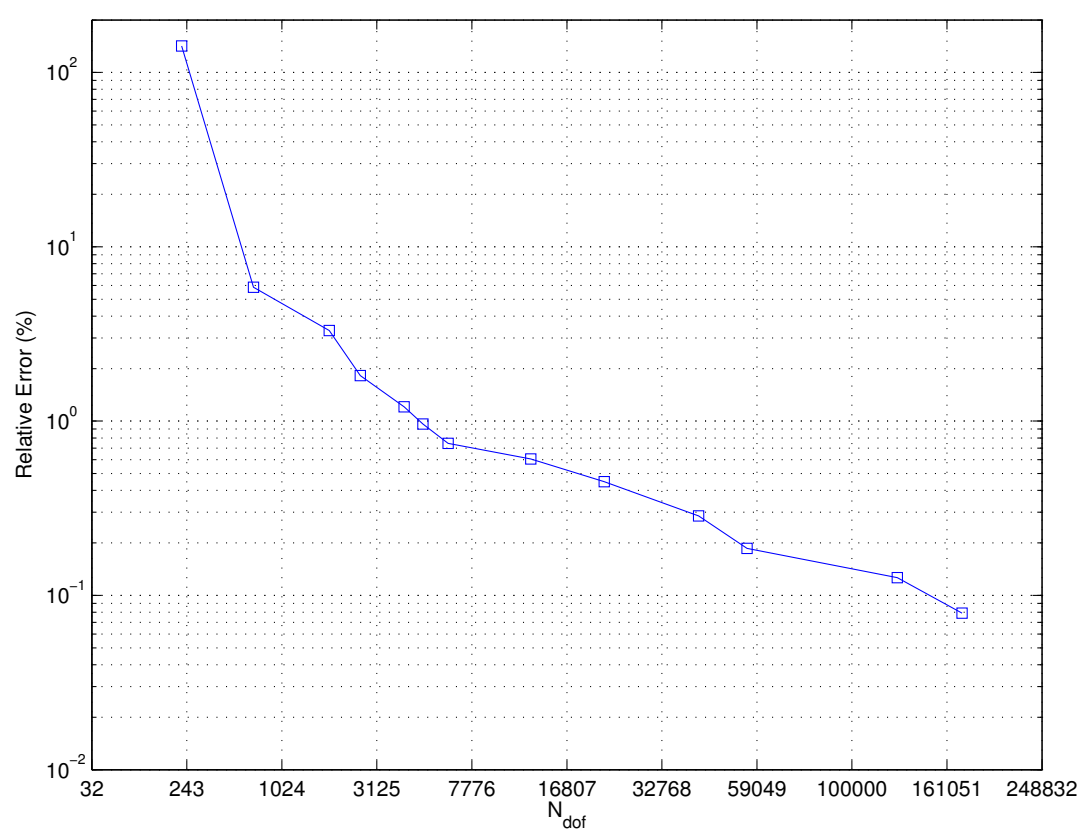

(a) Separation $d=0.5 a$

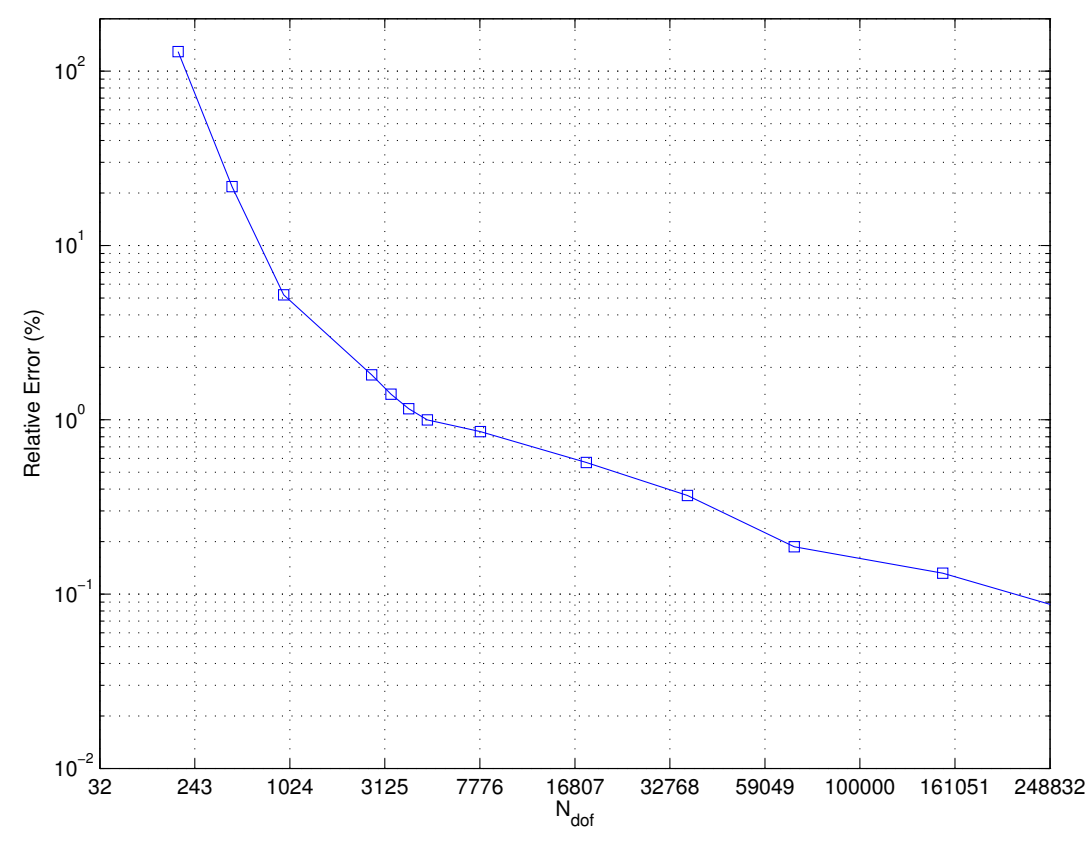

(b) Separation $d=0$

Figure 4: Convergence history for the cascade of H-plane and E-plane bends 


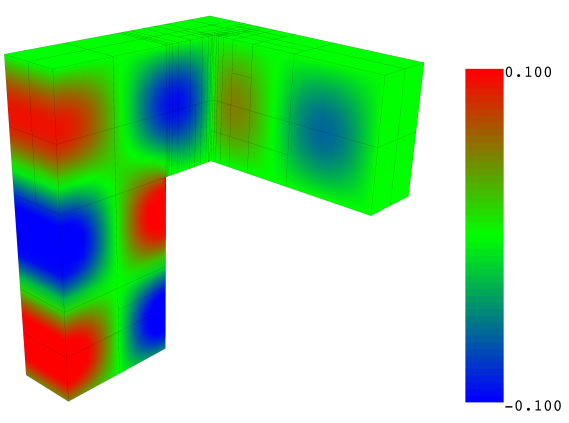

(a) Separation $d=0.5 a$

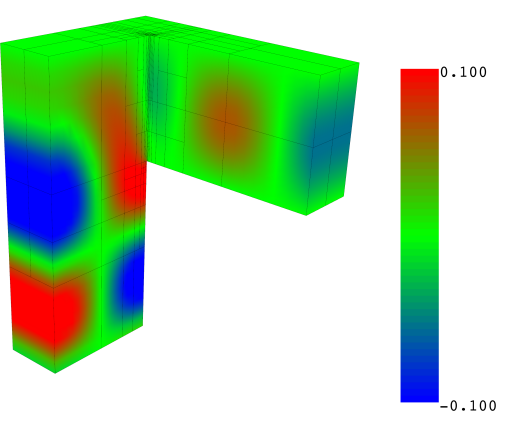

(b) Separation $d=0$

Figure 5: Real part of z-component of magnetic field for H-plane and E-plane bends (excitation by port 1).

Table 1: Scattering parameters for the cascade of H-plane and E-plane bends when $d=0.5 a$

\begin{tabular}{lcccc}
\hline & $\left|S_{11}\right|$ & $\left|S_{21}\right|$ & $\arg \left(S_{11}\right)$ & $\arg \left(S_{21}\right)$ \\
\hline Iter. 1 & 0.2552 & 0.7224 & $-73.307^{\circ}$ & $-83.489^{\circ}$ \\
Iter. 2 & 0.7437 & 0.6661 & $53.989^{\circ}$ & $91.961^{\circ}$ \\
Iter. 6 & 0.7368 & 0.6761 & $53.005^{\circ}$ & $89.757^{\circ}$ \\
Iter. 13 & 0.7359 & 0.6771 & $52.978^{\circ}$ & $89.755^{\circ}$ \\
MM & 0.7359 & 0.6771 & $52.983^{\circ}$ & $89.761^{\circ}$ \\
\hline
\end{tabular}

Table 2: Scattering parameters for the cascade of H-plane and E-plane bends when $d=0$

\begin{tabular}{lcccr}
\hline & $\left|S_{11}\right|$ & $\left|S_{21}\right|$ & $\arg \left(S_{11}\right)$ & $\arg \left(S_{21}\right)$ \\
\hline Iter. 1 & 0.8573 & 0.4414 & $164.455^{\circ}$ & $11.928^{\circ}$ \\
Iter. 2 & 0.6641 & 0.7464 & $-15.278^{\circ}$ & $-145.534^{\circ}$ \\
Iter. 6 & 0.6563 & 0.7545 & $-16.588^{\circ}$ & $-146.736^{\circ}$ \\
Iter. 13 & 0.6549 & 0.7557 & $-16.603^{\circ}$ & $-146.756^{\circ}$ \\
MM & 0.6550 & 0.7557 & $-16.607^{\circ}$ & $-146.751^{\circ}$ \\
\hline
\end{tabular}


Table 3: Scattering parameters for the EH-step

\begin{tabular}{lccrc}
\hline & $\left|S_{22}\right|$ & $\left|S_{21}\right|$ & $\arg \left(S_{22}\right)$ & $\arg \left(S_{21}\right)$ \\
\hline Iter. 1 & 0.41281 & 0.79590 & $131.029^{\circ}$ & $-044.294^{\circ}$ \\
Iter. 3 & 0.23060 & 0.97206 & $80.044^{\circ}$ & $-168.844^{\circ}$ \\
Iter. 9 & 0.21156 & 0.97733 & $85.398^{\circ}$ & $-167.060^{\circ}$ \\
Iter. 14 & 0.21185 & 0.97730 & $85.623^{\circ}$ & $-167.022^{\circ}$ \\
MM & 0.21193 & 0.97729 & $85.758^{\circ}$ & $-166.998^{\circ}$ \\
\hline
\end{tabular}

\subsection{Waveguide transition}

This structure contains a simultaneous change in the width and height of the waveguide. One waveguide section is geometrically centered with respect the other. The structure is shown in Fig. 6. It will be referred to as an "EHstep". This structure is used as transition to connect waveguide sections as in certain types of atennuators. Also, as mentioned in the Introduction, it is a component of more sophisticated devices, such as certain cavity filters.

The convergence history up to an error below $0.05 \%$ is shown in Fig. 7 . After an initial pre-asymptotic regime, the curve exhibits exponential convergence, as expected. The fourth mesh (see Fig. 8(a)) is displayed, since it is the first one when the adaptivity enters into the asymptotic regime. The ninth mesh (see Fig. 8(b)) delivers an error around 1\%. The initial mesh was designed to be as coarse as possible in terms of $h$ and with uniform $p=2$. An illustration of the magnetic field can be seen in Fig. 9; specifically, the real part of the "horizontal"-component. A strong (singular) field appears at the junction edges.

As in the previous case, $S_{21}=S_{12}$, and $S_{11} \neq S_{22}$, although $\left|S_{22}\right|=\left|S_{11}\right|$. The results for $S_{22}$ and $S_{21}$ are shown (for some of the $h p$ meshes) in Tab. 3. The scattering parameters computed with the $h p$-FEM method are compared with those obtained with a MM technique. Similar conclusions as those obtained for the previous case also hold here.

\subsection{Magic-T}

Fig. 10 describes the considered Magic-T structure. Some of its main properties in terms of its scattering parameters were detailed in the Introduction (see (1)). To illustrate the results delivered by the fully automatic 

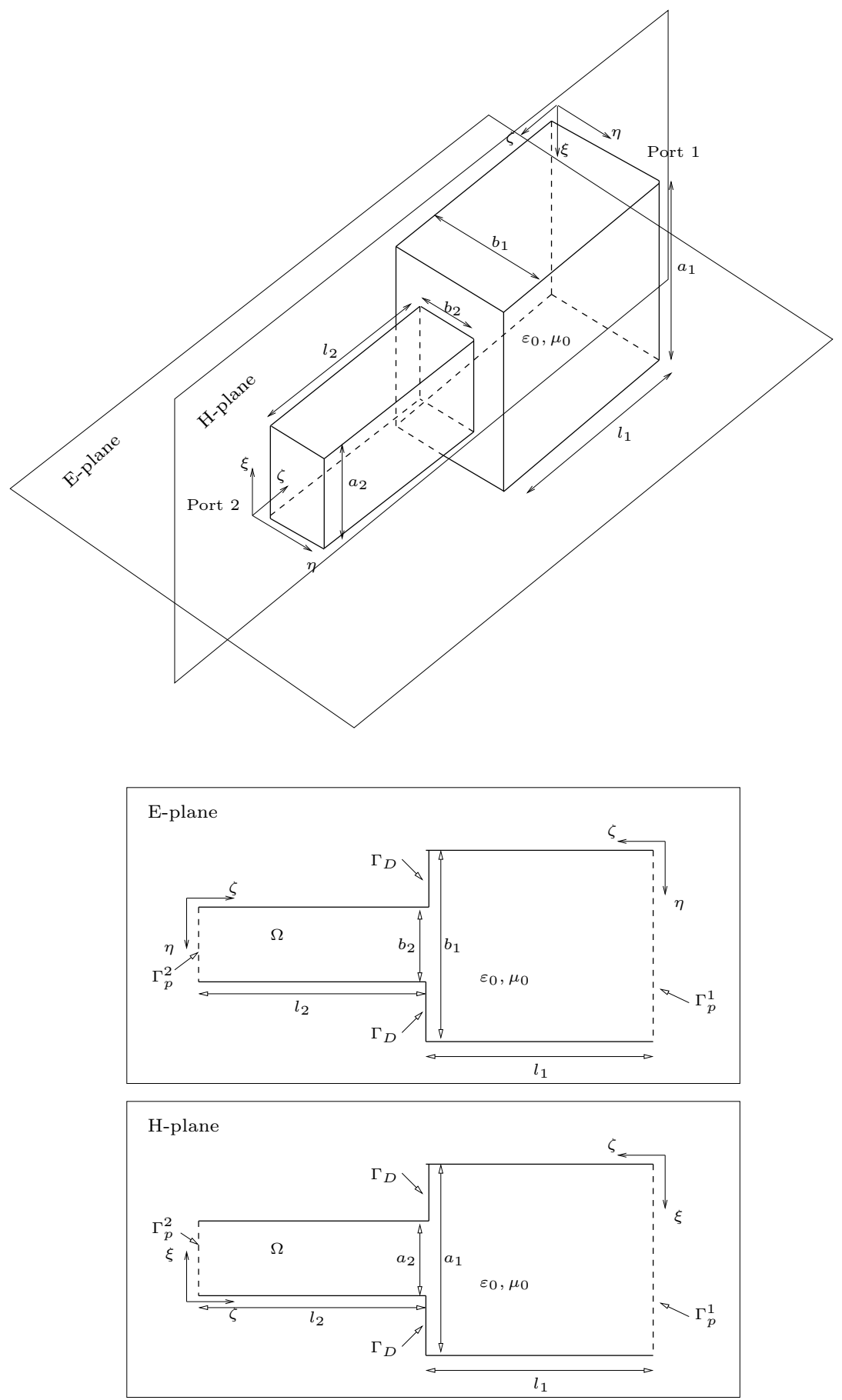

Figure 6: EH-step (change of width and height in waveguide: $a_{2}=0.8 a_{1}, b_{1}=$ $\left.a_{1} / 2, b_{2}=b_{1} / 2, l_{1}=l_{2} 1.5 a_{1}\right)$ 


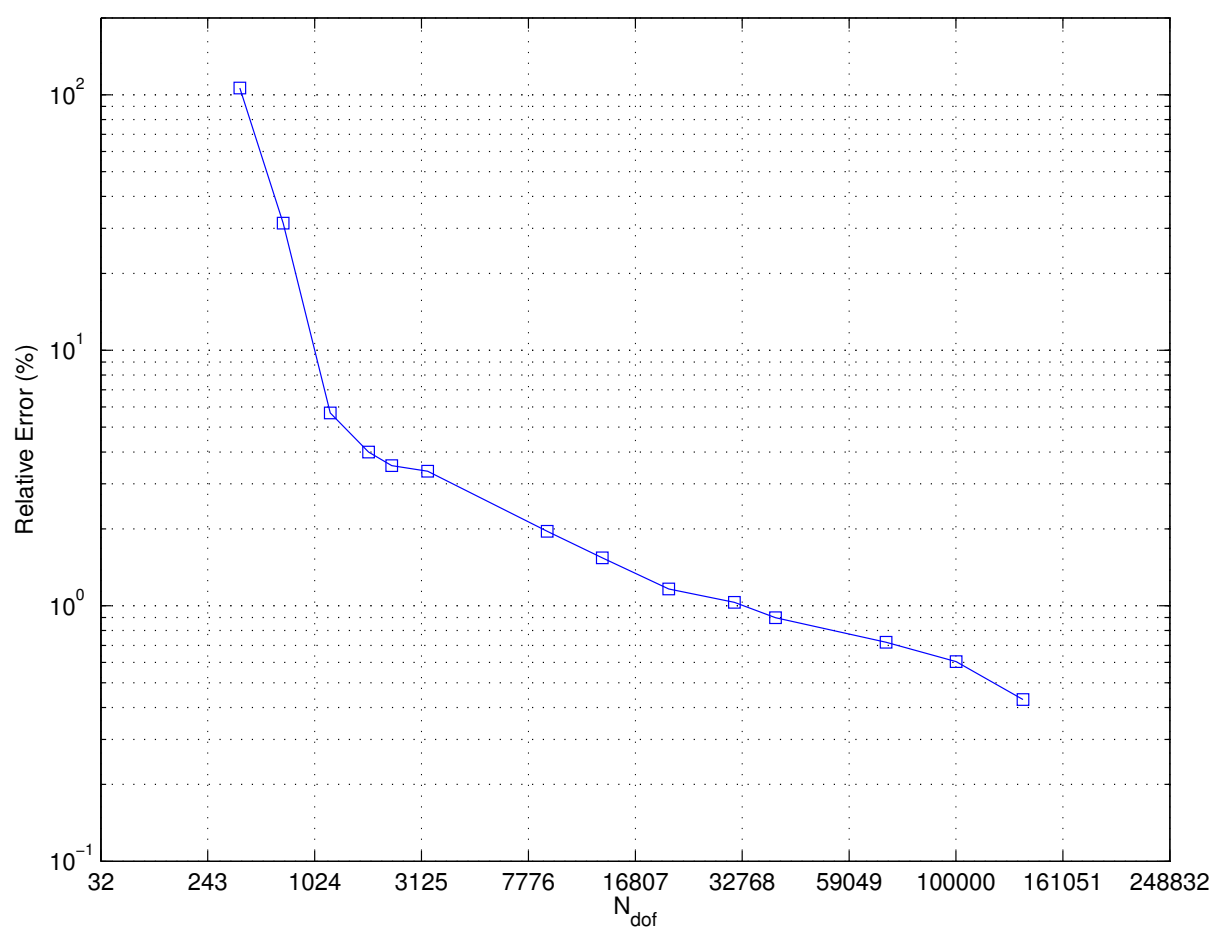

Figure 7: Convergence history for EH-step

$h p$-adaptivity applied to this structure, we display in Fig. 11 two meshes corresponding to the 3rd and 13th iteration of the adaptive procedure for the Magic-T structure excited by port 1. As expected, we observe heavy $p$-refinements in those areas of the domain where solution is smooth (away from the junctions) and additional $h$-refinements where a singular behavior of some components of the solution is expected (close to the junctions).

This adaptive strategy delivers exponential convergence rates, as exhibited in Fig. 12, where we display the convergence history (up to an error around $0.3 \%$ ) for the Magic-T structure excited by ports 3 and 4 .

The tenth mesh delivered by the $h p$-adaptivity when exciting port 3 is shown in Fig. 13 (left panel). The ninth mesh delivered when exciting port 4 is displayed in Fig. 13 (right panel). Both meshes correspond to an energy error around 1\%. The initial mesh was selected to be as coarse as possible in terms of $h$ and with uniform $p=2$. The large difference of the meshes at this error level can be explained as follows. When exciting port 3, the T-junction of the H-plane is excited (ports 1,2,3). And when port 4 is excited, the 

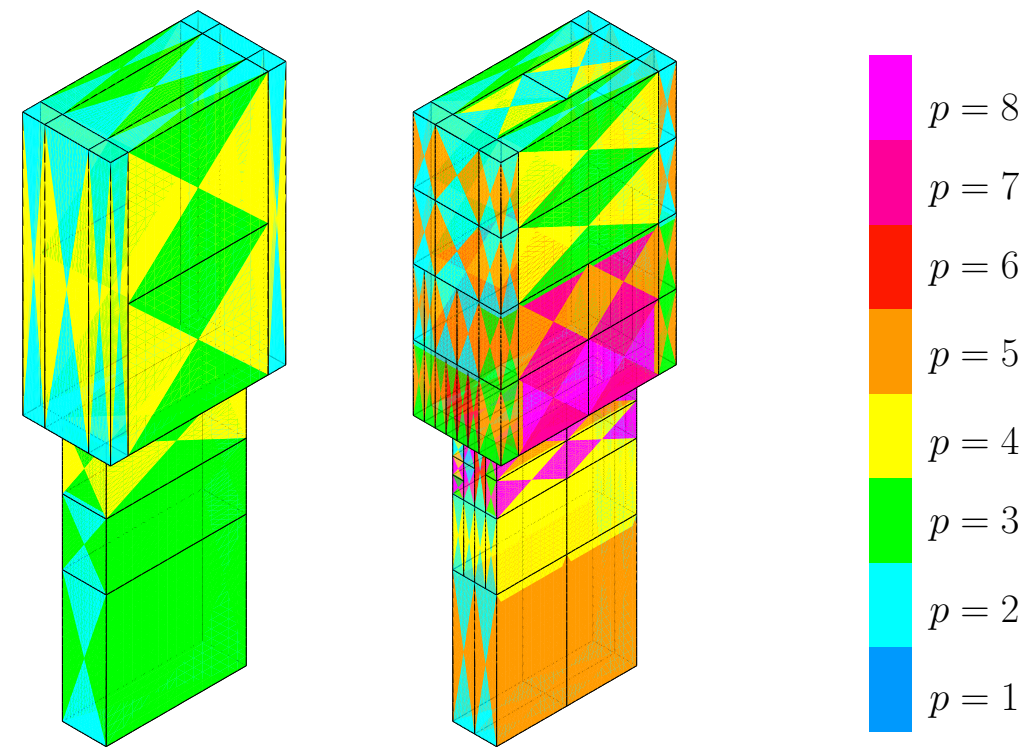

(a) Mesh of 3rd iteration (b) Mesh of 9th iteration

Figure 8: Meshes of the EH-step. Different colors indicate different polynomials orders of approximation.

T-junction of the E-plane is excited (ports 1,2,4). The field of the H-plane T-junction is quite different to the one of the E-plane T-junction and so are the meshes delivered by the adaptivity.

The isolation between ports 3 and 4 is illustrated in Fig. 14, in which the magnitude of the magnetic field in the structure when exciting port 3 is displayed. Specifically, the component of the displayed magnetic field corresponds to the longitudinal component of port 3 and the transverse component of ports 1 and 2. A zero solution is observed in port 4. Note the stationary wave pattern in waveguide of port 3 due to existence of a non-negligible reflected wave in addition to the incident wave. No stationary wave is observed in the output waveguides of ports 1 and 2 as there is only one wave propagating outward on each of the transmitted ports.

As the structure has four ports, there is a maximum of sixteen $S$-parameters to be computed. However, by reciprocity $S_{j i}=S_{i j}$. In addition, the symmetry of the Magic-T forces other relations, e.g., $S_{41}=S_{42}$. In summary, the 

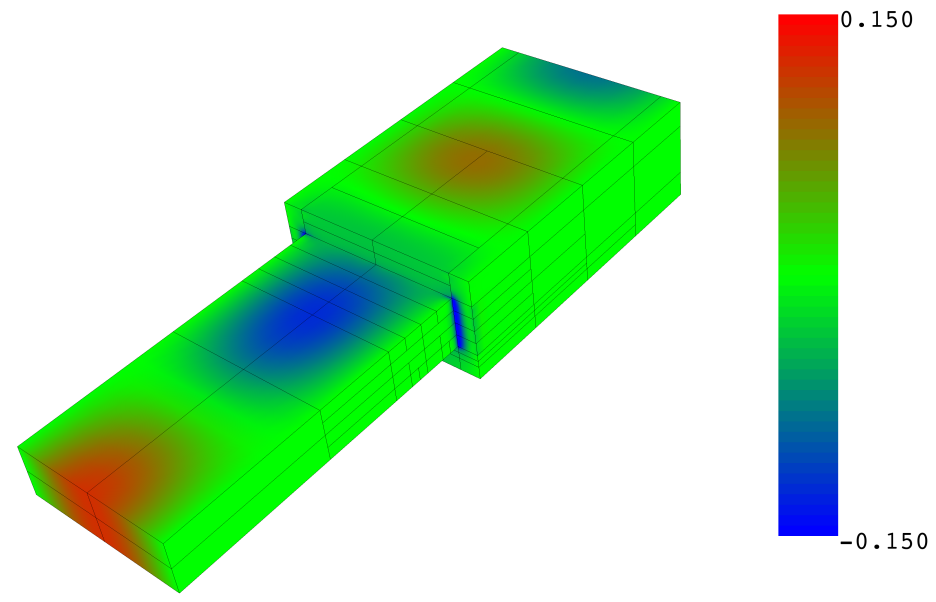

Figure 9: Real part of x-component of magnetic field for the EH-step (excitation by port 2 ).

$[S]$ matrix can be defined in terms of only seven parameters as follows:

$$
\left\{\begin{array}{l}
b_{1} \\
b_{2} \\
b_{3} \\
b_{4}
\end{array}\right\}=\left[\begin{array}{llll}
S_{11} & S_{21} & S_{31} & S_{41} \\
S_{21} & S_{11} & S_{31} & S_{41} \\
S_{31} & S_{31} & S_{33} & S_{43} \\
S_{41} & S_{41} & S_{43} & S_{44}
\end{array}\right]\left\{\begin{array}{l}
a_{1} \\
a_{2} \\
a_{3} \\
a_{4}
\end{array}\right\}
$$

The values computed by $h p$-FEM of some of the $S$-parameters obtained when exciting ports 3 and 4 are shown in Tab. 4 . Those values are compared with the corresponding ones obtained with a MM technique. Only the magnitudes are shown; the $S_{i j}$ phase difference between the results delivered by the $h p$-FEM and MM is around $0.01^{\circ}$. Only four significant digits are shown in the table as the MM results are presumed to have no more than four digits of accuracy. Note that $S_{43}=0$. This is the result obtained numerically (close to machine precision) with MM. Similar values are provided by the hp-FEM. Note that scattering matrix does not correspond to a scattering matrix of a perfectly compensated Magic-T, since our waveguide structure has intentionally been left uncompensated. 


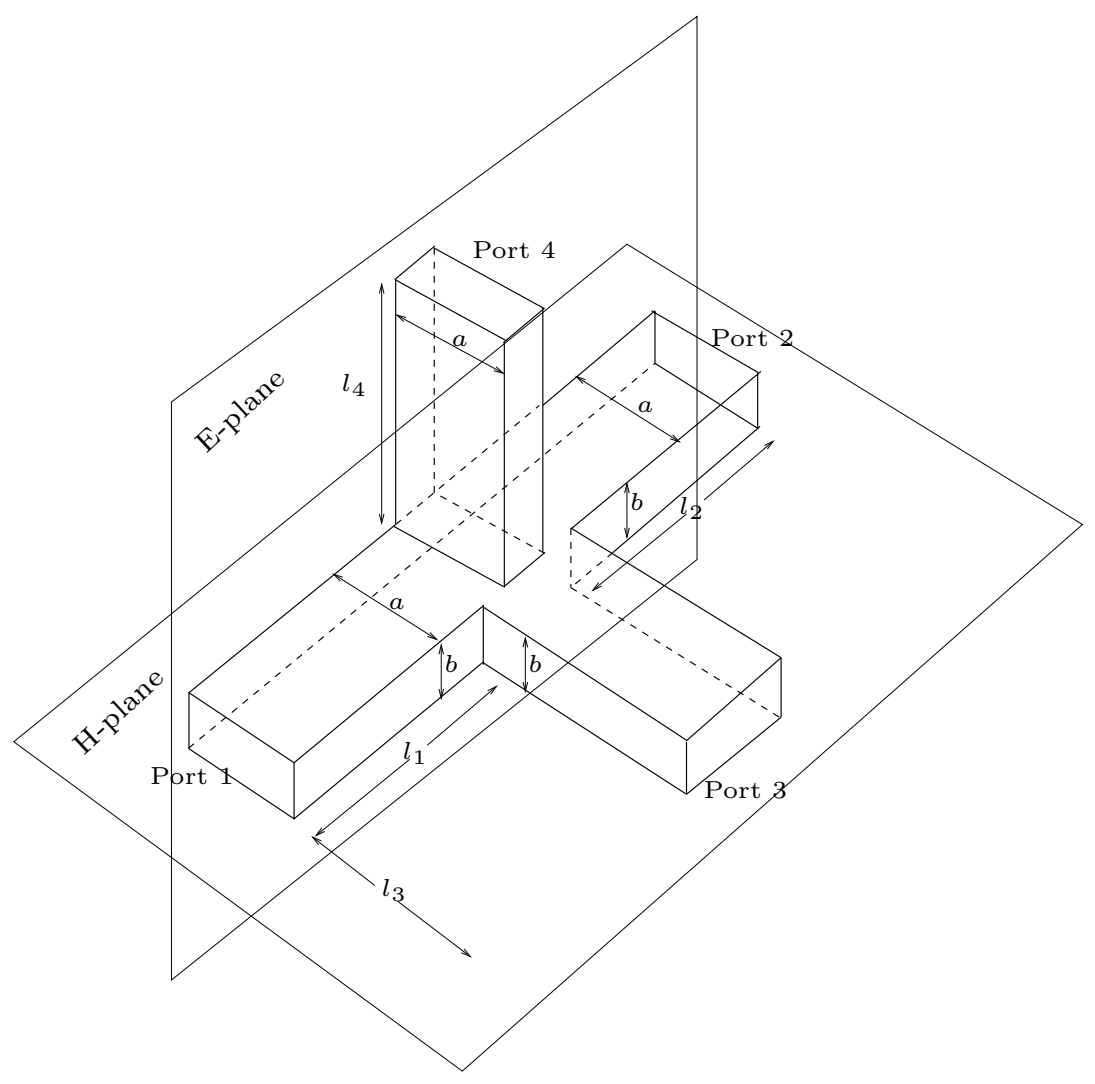

Figure 10: Geometry of a Magic-T waveguide $\left(b=a / 2, l_{1}=l_{2}=l_{3}=l_{4}=1.5 a\right)$

Table 4: Magnitude of scattering parameters for the Magic-T.

\begin{tabular}{lccccccc}
\hline & $\left|S_{11}\right|$ & $\left|S_{21}\right|$ & $\left|S_{31}\right|$ & $\left|S_{41}\right|$ & $\left|S_{33}\right|$ & $\left|S_{43}\right|$ & $\left|S_{44}\right|$ \\
\hline Iter. 1 & 0.0975 & 0.4256 & 0.5687 & 0.3525 & 0.4202 & $10^{-15}$ & 0.4731 \\
Iter. 3 & 0.1099 & 0.5727 & 0.5883 & 0.6270 & 0.5535 & $3 \times 10^{-15}$ & 0.4604 \\
Iter. 10 & 0.0536 & 0.5086 & 0.5858 & 0.6291 & 0.5601 & $10^{-13}$ & 0.4565 \\
Iter. 12 & 0.0524 & 0.5083 & 0.5858 & 0.6291 & 0.5601 & $3 \times 10^{-15}$ & 0.4566 \\
MM & 0.0521 & 0.5083 & 0.5859 & 0.6290 & 0.5599 & $10^{-15}$ & 0.4568 \\
\hline
\end{tabular}



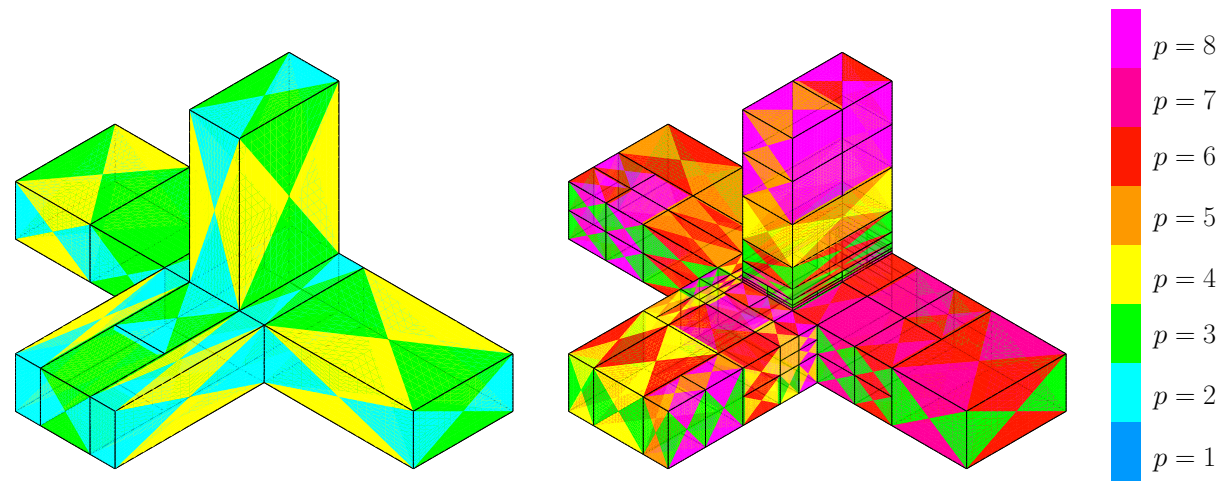

Figure 11: Two meshes produced by the self-adaptive $h p$-refinement strategy for the Magic-T problem when excited by port 1 . Left panel: Mesh after 3 iterations. Right panel: Mesh after 13 interactions. Different colors indicate different polynomials orders of approximation.

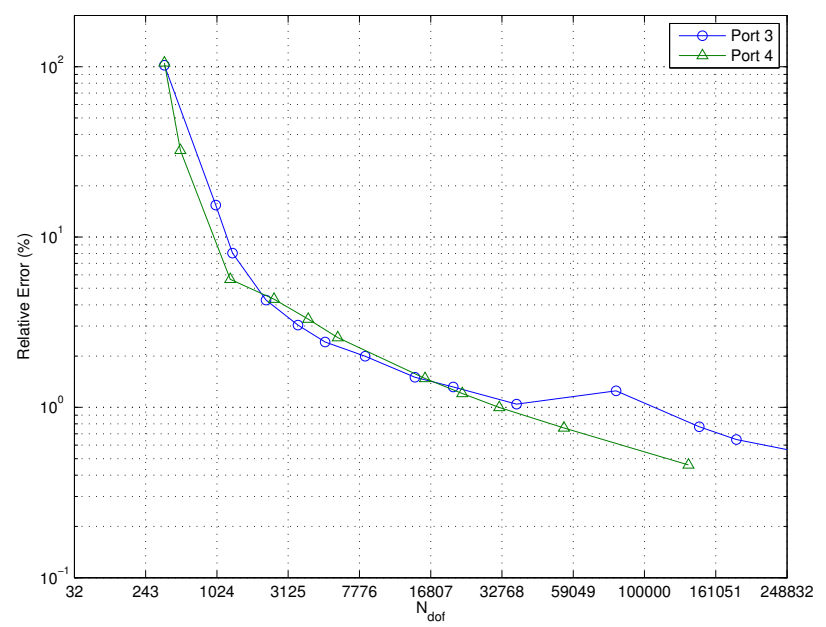

Figure 12: Convergence history for Magic-T. 

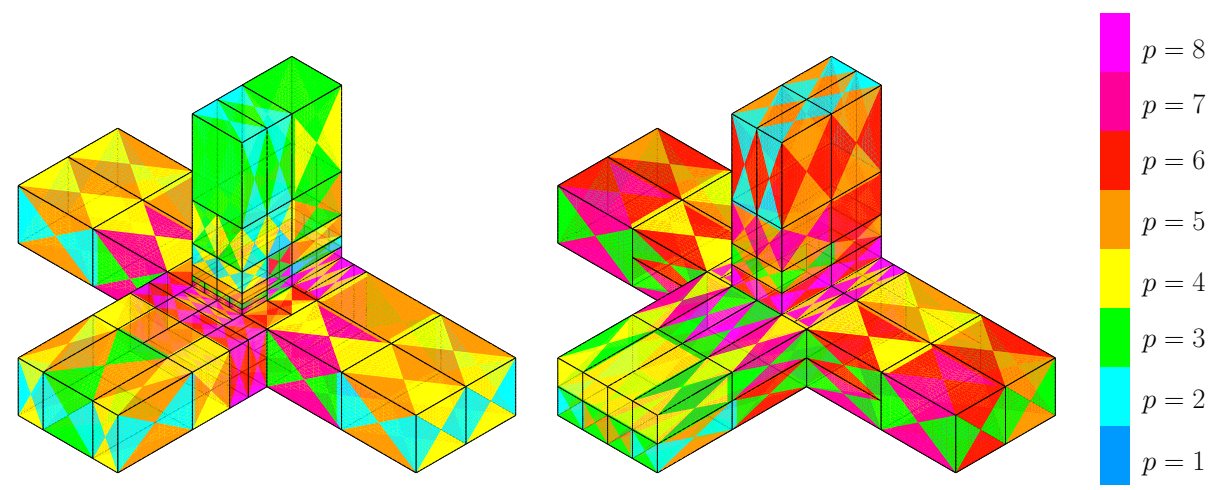

Figure 13: Left panel: Mesh of 10th iteration for Magic-T (excitation by port 3). Right panel: Mesh of 9 th iteration for Magic-T (excitation by port 4).

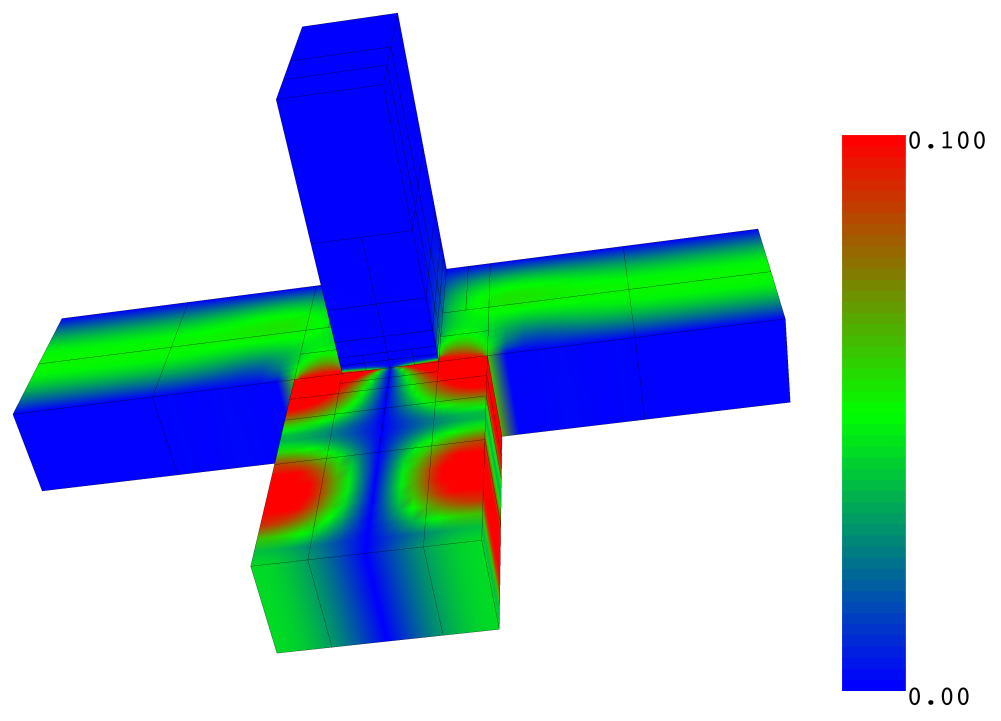

Figure 14: Magnitude of $\mathrm{x}$-component of magnetic field for Magic-T (excitation by port 3$)$. 


\section{Conclusions}

An $h p$-adaptive FE strategy has been employed for the simulation of several relevant waveguide structures such as a bend, a step, and a magic-T. Computation of the scattering matrix used to characterize the electromagnetic behavior of these structures has been implemented as a post-processing of the solution. Grids delivered by the $h p$-adaptive strategy indicate an optimal (or quasi-optimal) distribution of element sizes $h$ and polynomial orders of approximation $p$ throughout the computational grid. These results have been confirmed by the excellent convergence curves, and the final simulations which have a comparable accuracy to those obtained with semianalytical methods. At the same time, the $h p$-FEM maintains the flexibility

of any FEM, which can easily account for different geometries, materials, and construction or design artifacts.

\section{References}

[1] W. Tyrell, Hybrid circuits for microwaves, Proceedings of the IRE 35 (11) (1947) 1294-1306.

[2] J. M. Reiter, F. Arndt, Efficient FDTD/Matrix-Pencil method for the full-wave scattering parameter analysis of waveguiding structures, IEEE Transactions on Microwave Theory and Techniques 44 (12) (1996) 24502456.

[3] G. Conciauro, M. Guglielmi, R. Sorrentino, Advanced Modal Analysis: CAD Techniques for Waveguide Components and Filters, John Wiley \& Sons, Inc., 2000.

[4] J. M. Rebollar, J. Esteban, J. E. Page, Fullwave analysis of three and four-port rectangular waveguide junctions, IEEE Transactions on Microwave Theory and Techniques 42 (2) (1994) 256-263.

[5] F. Alessandri, M. Mongiardo, R. Sorrentino, Computer-aided design of beam forming networks for modern satellite antennas, IEEE Transactions on Microwave Theory and Techniques 40 (6) (1992) 1117-1127.

[6] T. Shen, H.-T. Hsu, K. A. Zaki, A. E. Atia, T. G. Dolan, Full-wave design of canonical waveguide filters by optimization, IEEE Transactions on Microwave Theory and Techniques 51 (2) (2003) 504-511. 
[7] V. de la Rubia, J. Zapata, Microwave circuit design by means of direct decomposition in the finite-element method, IEEE Transactions on Microwave Theory and Techniques 55 (7) (2007) 1520-1530.

[8] M. Mongiardo, R. Sorrentino, Efficient and versatile analysis of microwave structures by combined mode matching and finite difference methods, IEEE Microwave and Guided Wave Letters 3 (8) (1993) 241243.

[9] P. Arcioni, Fast evaluation of modal coupling coefficients of waveguide step discontinuities, IEEE Microwave and Guided Wave Letters 6 (6) (1996) 232-234.

[10] F. Arndt, J. Brandt, V. Catina, J. Ritter, I. Rullhusen, J. Dauelsberg, U. Hilgefort, W. Wessel, Fast CAD and optimization of waveguide components and aperture antennas by hybrid MM/FE/MoM/FD methods state-of-the-art and recent advances, IEEE Transactions on Microwave Theory and Techniques 52 (1) (2004) 292-305.

[11] F. Ihlenburg, I. Babuška, Finite element solution of the Helmholtz equation with high wave number. I: The $h$-version of the FEM, Computer and Mathematics with Applications 30 (9) (1995) 9-37.

[12] F. Ihlenburg, I. Babuška, Finite element solution of the Helmholtz equation with high wave number. II: The $h-p$-version of the FEM, SIAM Journal of Numerical Analysis 34 (1) (1997) 315-358.

[13] L. E. García-Castillo, D. Pardo, I. Gómez-Revuelto, L. F. Demkowicz, A two-dimensional self-adaptive $h p$-adaptive finite element method for the characterization of waveguide discontinuities. Part I: Energynorm based automatic $h p$-adaptivity, Computer Methods in Applied Mechanics and Engineering 196 (49-52) (2007) 4823-4852, doi:10.1016/j.cma.2007.06.024.

[14] L. F. Demkowicz, A. Buffa, $H^{1}, H$ (curl) and $H$ (div)-conforming projection-based interpolation in three dimensions. Quasi optimal $p$ interpolation estimates, Computer Methods in Applied Mechanics and Engineering 195 (24) (2006) 4816-4842, see also ICES Report 04-22.

[15] M. Paszynski, L. F. Demkowicz, Parallel, fully automatic $h p$-adaptive 3D finite element package, Tech. Rep. 33, ICES (Aug. 2005). 
[16] J. P. Kurtz, Fully automatic $h p$-adaptivity for acoustic and electromagnetic scattering in three dimensions, Ph.D. thesis, CAM Ph.D. Program, ICES, University of Texas at Austin, (Ph.D. proposal) (Sep. 2005).

[17] J. Kurtz, L. F. Demkowicz, A fully automatic $h p$-adaptivity for elliptic PDEs in three dimensions, Computer Methods in Applied Mechanics and Engineering 196 (2007) 3534-3545, doi:10.1016/j.cma.2006.10.053.

[18] L. Demkowicz, J. Kurtz, D. Pardo, M. Paszynski, W. Rachowicz, A. Zdunek, Computing with $h p$ Finite Elements. II Frontiers: Three Dimensional Elliptic and Maxwell Problems with Applications, Chapman \& Hall/CRC Press, Taylor and Francis, 2008.

[19] L. Demkowicz, Computing with $h p$ Finite Elements. I. One- and TwoDimensional Elliptic and Maxwell Problems, Chapman \& Hall/CRC Press, Taylor and Francis, 2007.

[20] F. Ihlenburg, I. Babuška, Dispersion analysis and error estimation of Galerkin finite element methods for Helmhotz equation, International Journal for Numerical Methods in Engineering 38 (1995) 3745-3774.

[21] L. F. Demkowicz, Encyclopedia of Computational Mechanics, John Wiley \& Sons, Inc., 2004, Ch. "Finite Element Methods for Maxwell Equations".

[22] L. F. Demkowicz, A. Buffa, $H^{1}, H$ (curl) and $H$ (div)-conforming projection-based interpolation in three dimensions. Quasi optimal $p$ interpolation estimates, Computer Methods in Applied Mechanics and Engineering 194 (2-5) (2005) 267-296.

[23] W. Rachowicz, D. Pardo, L. F. Demkowicz, Fully automatic $h p-$ adaptivity in three dimensions, Computer Methods in Applied Mechanics and Engineering 195 (37-40) (2006) 4186-4842.

[24] W. Gui, I. Babuška, The $h, p$ and $h-p$ versions of the finite element method in 1 dimension - Part I. The error analysis of the $p$-version, Numerische Mathematik 49 (1986) 577-612.

[25] W. Gui, I. Babuška, The $h, p$ and $h-p$ versions of the finite element method in 1 dimension - Part II. The error analysis of the $h$ - and $h-p$ versions, Numerische Mathematik 49 (1986) 613-657. 
[26] W. Gui, I. Babuška, The $h, p$ and $h-p$ versions of the finite element method in 1 dimension - Part III. The adaptive $h-p$ version, Numerische Mathematik 49 (1986) 659-683.

[27] I. Babuška, B. Guo, Regularity of the solutions of elliptic problems with piecewise analytic data. Part I. Boundary value problems for linear elliptic equation of second order, SIAM Journal of Mathematical Analysis 19 (1) (1988) 172-203.

[28] I. Babuška, B. Guo, Regularity of the solution of elliptic problems with piecewise analytic data. II: The trace spaces and application to the boundary value problems with nonhomogeneous boundary conditions, SIAM Journal of Mathematical Analysis 20 (4) (1989) 763-781.

[29] I. Babuška, B. Guo, Approximation properties of the $h p$-version of the finite element method, Computer Methods in Applied Mechanics and Engineering 133 (1996) 319-346.

[30] C. Schwab, $p$ - and $h p$ - Finite Element Methods. Theory and Applications in Solid and Fluid Mechanics, Oxford University Press, 1998. 